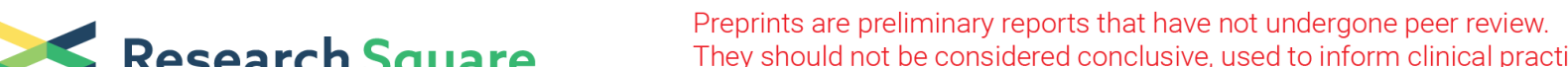 $\begin{array}{ll}\text { Research Square } & \text { They should not be considered conclusive, used to inform clinical practice, } \\ \text { or referenced by the media as validated information. }\end{array}$
}

\section{Structural network topology correlates to cognitive impairment and iPTH level in End-stage renal disease patients with peritoneal dialysis}

\section{Longsheng Wang}

the Second Hospital of Anhui Medical University

Liwei Zou

Anhui Medical University

Hanqiu Wu

the Second Hospital of Anhui Medical University

Yanqi Shan

the Second Hospital of Anhui Medical University

Ru Zhao

the Second Hospital of Anhui Medical University

\section{Guiling Liu}

the Second hospital of Anhui Medical University

\section{Fangbiao Tao}

Anhui Medical University

\section{Suisheng Zheng}

Ping An Healthcare Diagnostics Center

Xijun Gong ( $\square$ gongxj0306@sina.com )

the Second Hospital of Anhui Medical University

\section{Research article}

Keywords: end-stage renal disease, secondary hyperparathyroidism, structural connectivity, topological organization, network

Posted Date: August 13th, 2019

DOI: https://doi.org/10.21203/rs.2.9318/v5

License: (c) (1) This work is licensed under a Creative Commons Attribution 4.0 International License.

Read Full License 


\section{Abstract}

Background: The burden of cognitive impairment in patients with end-stage renal disease (ESRD) undergoing peritoneal dialysis has received more attention lately. It associates with hospitalization, mortality and poorer quality of life. We aimed to assess the topological alterations of the brain white matter structural network in ESRD and the correlation between network metrics with Montreal Cognitive Assessment scores and clinical data. Methods: The study included 25 ESRD patients with secondary hyperparathyroidism (SHPT group), 25 patients without SHPT (Non-SHPT group) and 25 healthy controls ( $\mathrm{HC}$ group) of comparable age and sex. Montreal Cognitive Assessment was used to assess the cognitive function. The white matter (WM) structural network was constructed by diffusion tensor imaging (DTI) technique, and then we used graph theoretical approaches to detect changes in the global and regional properties of the WM networks in these participants through deterministic tractography method. Results: ESRD patients showed cognitive impairment compared to HC, and the SHPT patients had lower cognitive scores than the Non-SHPT patients. The global topological organization and local efficiency of the WM network was significantly disrupted in the SHPT but not in the Non-SHPT patients compared with the HC group. Moreover, lower regional efficiency was found in the ESRD patients, mainly distributed in the frontal and parietal cortices. In addition, an association was observed between intact parathyroid hormone (iPTH), shortest path length and cognitive impairment, and the iPTH level was negatively correlated with small-worldness by two indexes, the normalized clustering coefficient and the normalized shortest path length. Conclusion: The present study indicated that the brain structural connectome in ESRD patients with high iPTH levels was disrupted in association with cognitive impairment and it is a potential connectome-based biomarker for early detection.

\section{Background}

Chronic kidney disease (CKD) is an important issue that is of widespread public concern. There are approximately 571000 patients with end-stage renal disease (ESRD) receiving treatment in the United States, and nearly from 270000 to 550000 patients with ESRD undergo hemodialysis in China from 2012 to 2015 year [1-3]. Cognitive impairment has received more attention recently in ESRD patients because it has a prevalence of approximately $30 \%-60 \%[1,4,5]$. Cognitive impairment is associated with adverse outcomes and a decreased quality of life [6,7].

Cerebrovascular disease, anemia, uremic toxins, and secondary hyperparathyroidism (SHPT) have been reported to be major causes of cognitive impairment in CKD patients [8-11]. SHPT is characterized by high parathyroid hormone (PTH) levels, low vitamin D levels and normal or low calcium levels. There is some evidence that PTH levels may correlate with cognitive impairment and the risk of dementia $[12,13]$. However, the pathophysiology of cognitive impairment in ESRD patients with SHPT is still unclear.

Neuroimaging has been established as a reliable tool to assess the neuropathologic mechanisms of cognitive impairment in ESRD patients [14, 15]. Resting-state functional magnetic resonance imaging (rsfMRI) showed that ESRD patients have lower amplitude of low frequency fluctuation (ALFF) values and 
abnormal brain functional connectivity in default mode network (DMN) regions when compared with healthy controls [16]. Some studies have found the diffusion tensor imaging (DTI) technique can reconstruct whole brain white matter (WM) connectivity $[17,18]$. Graph theoretical analysis suggested that WM networks characterize many important topological organizations, like small-worldness [19]. To date, no studies have been conducted to compare topological differences in WM networks between SHPT and non-SHPT ESRD patients.

In the current study, we hypothesized that the topological organization of the WM network was changed in ESRD patients undergoing peritoneal dialysis, and we further detected a relationship between the neurocognitive scores and clinical biomarkers with network metrics. To test the hypothesis, we included different physiological data from 50 patients with ESRD and 25 healthy controls, including cognitive tests, laboratory tests and DTI data. By using DTI track diagram and graph theory, we compared the changes of WM network topology property in ESRD patients with or without SHPT and healthy controls. Then, correlation analyses among network topographical organizations, global cognitive scores and clinical biomarkers were conducted.

\section{Methods And Materials}

\section{Participants}

The present study was approved by the Ethics Committee of the Second Hospital of Anhui Medical University. Written informed consent was provided from all participants. Fifty peritoneal dialysis ESRD patients ( 25 patients with SHPT, 14 males and 11 females, mean age $42.20 \pm 7.53$ years (SHPT group); 25 patients without SHPT, 15 males and 10 females, mean age $41.96 \pm 6.17$ years (Non-SHPT group)) were enrolled at the Nephrology Department in our hospital from January 2017 to July 2018. Inclusion criteria for patients as followings: (a) ESRD patients were diagnosed by (GRE (estimated glomerular filtration rate) $\leq 15 \mathrm{~mL} / \mathrm{min} / 1.73 \mathrm{~m}^{2}$ ), (b) receiving regular peritoneal dialysis three times a week at our hospital, (c) SHPT for iPTH level $>600 \mathrm{pg} / \mathrm{ml}$ (9 times of normal upper level). 25 healthy controls (HC group, 12 males and 13 females, mean age $41.80 \pm 7.15$ years) were recruited. The healthy controls had no kidney disease and did not undergo laboratory tests.

All subjects were right-handed, and more than 18 years old. All participants received the neuropsychological test and MRI examination on the same day, receiving regular hemodialysis three times a week in our hospital. Exclusion criteria were as follows: (1) obvious encephalopathy (cerebrovascular disease) revealed by clinical or imaging, (2) any history of drug/alcohol abuse, (3) leukoencephalopathy on a previous MRI, and (4) MRI contraindications.

\section{Neuropsychological test}

Before the MRI scan, we used the Montreal Cognitive Assessment (MoCA) to assess the participants' global cognitive abilities. A score less than 26 was defined as a diagnosis of cognitive impairment [20]. 


\section{Laboratory test}

Serum creatinine and urea levels within 24 hours prior to the MRI scan were conducted to evaluate renal function of all patients.

\section{MRI Acquisition}

MR imaging data were acquired in a Siemens Verio 3.0T MR scanner using an 8-channel coil. Use foam padding to reduce head motion. MRI sequence include 3D T1 weighted structural sagittal images ( $\mathrm{TE}=2.98 \mathrm{~ms}, \mathrm{TR}=1900 \mathrm{~ms}$, thickness $=1 \mathrm{~mm}$, voxel size $=1 \times 1 \times 1 \mathrm{~mm}^{3}, \mathrm{FOV}=256 \times 256,176$ slices $)$ and diffusion weighted images ( 30 directions, $T E=84 \mathrm{~ms}, T R=8400 \mathrm{~ms}$, slice thickness $=3 \mathrm{~mm}, F O V=256 \times 256$, $\mathrm{b}=0,1000 \mathrm{~s} / \mathrm{mm}^{2}$, no gap) in all subjects. All subjects were told to keep their eyes closed and remain awake within the MRI scanning process.

\section{Data Processing}

Data preprocessing and network construction are accomplished by using PANDA toolbox (www.nitrc.org/projects/panda) [21]. Briefly, the image preprocessing procedure included format conversion from DICOM to NIFTI format, BET (skull removal), extract brain tissue and structure, eddy current and motion artifact correction, calculation of fractional anisotropy (FA) and diffusion tensor tractography. Fiber tracts were terminated if the FA value was lower than 0.2 , or exceed an angular threshold of $45^{\circ}$.

\section{WM network construction}

Network node definition. The automated anatomical labeling (AAL) atlas [22] were used to parcel 90 cortical and subcortical regions (45 for each brain hemisphere) to define the network nodes [19] (Table 1, Figure 1).

Network edge definition. To define the edges of the structural network, we selected a threshold value for the fiber bundles with two end points located in both two regions. To remove spurious connections, we used a minimum of three fibers as a threshold [23]. We used fiber number (FN) of the connected fibers between two regions for the weights of the network edges. Finally, for each participant, the FN weighted $90 \times 90$ matrix structural networks were constructed [24] (Figure 1).

\section{Network analysis}

We performed by GRETNA (www.nitrc.org/projects/gretna) toolbox to analyze the WM network topological properties [25], included clustering coefficient (Cp), shortest path length (Lp), small-worldness $(\sigma)$ (normalized clustering coefficient $(\gamma)$ and normalized shortest path length $(\lambda)$, local efficiency (Eloc), and global efficiency (Eg). The Cp of a network is the average of the clustering coefficient of each node in the network, which indicates the local efficiency for the transformation of the information. The Lp of the 
entire network refers to the average shortest travel distance across all nodes and indicates the most efficiency information transfer between the two nodes.

In order to test $\gamma$ and $\lambda$ in this study, 100 matched random networks are generated by Markov-chain Monte Carlo method. These networks have the same number of nodes, edges and degree distributions, but retain the weighted distribution of the real network. We computed the $\mathrm{y}(\mathrm{y}=\mathrm{Cp} / \mathrm{Cp}$ random $)$ and $\lambda$ $\left(\lambda=\mathrm{Lp} / \mathrm{L} \mathrm{p}_{\text {random }}\right)$, where $C \mathrm{p}_{\text {random }}$ and $\mathrm{L} \mathrm{p}_{\text {random }}$ are the average clustering coefficient and shortest path length over the random network, respectively. A small world network should meet the $y>1$ and $\lambda \approx 1$.

Eg is the average of the inverse of the shortest path length of all node pairs in the network and usually reflects the ability of the network in parallel information processing. Eloc is the average of the global efficiency of the community neighboring all nodes in the network and represents the fault tolerance level of the network.

\section{Identification of Hubs}

Hubs of networks are key nodes that are estimated by different methods [26, 27]. Consistent with previous study [28], we identify the hub regions by regional efficiency. The hubs defined with regional efficiency at least one SD greater than the mean nodal efficiency of the network.

\section{Statistical analysis}

Demographic factors and clinical data were analyzed by SPSS 23.0 (SPSS, Chicago, IL, USA). We used mean \pm SD for continuous variables, and frequencies for categorical variables in descriptive analysis. The differences among three groups including age, education and neuropsychological tests were analyzed with one-way analysis of variance (ANOVA). Post hoc pairwise comparisons were then performed using $t$ tests. The sex data were analyzed by the chi-square test. In addition, two-sample t test was used to compare the clinical data between two groups for normality and homoscedasticity of the distributions, otherwise the Mann-Whitney U test was used.

For group effects in global and regional network metrics, comparisons were conducted among the three groups by one-way ANOVA and Nonparametric test (Kruskal-Wallis Test) for normal distribution and abnormal distribution, respectively. Levels of significance were set at $p<0.05$ and a Bonferroni correction for multiple testing was applied for group differences $(a=0.05 / 3=0.017)$. We also detected the relationship between network metrics, neuropsychological test scores and the clinical index by partial correlation analysis. To investigate the correlation between the neuropsychological test scores and specific brain regions, the nodes with a significant group differences were taken.

\section{Results}

\section{Neuropsychological and Clinical Assessments}


The demographic, neuropsychological, and clinical data of the patients are summarized in Table 2. Differences in age, sex, and education level between groups were not significant $(p>0.05)$. No significant differences were found in disease duration or blood biochemistry between the two patient groups $(p>0.05)$ except for intact parathyroid hormone (iPTH) levels $(p<0.01)$. For the neuropsychological test, there were significantly decreased global cognition scores among both the SHPT and Non-SHPT patients compared with the healthy controls $(p<0.01)$.

\section{Small-Worldness}

All three groups showed a typical small-world organization of structural networks expressed as $\gamma>1$ and $\lambda \approx 1$ (Figure 2). Compared with the healthy controls, the SHPT patients had a significantly decreased Cp $(p=0.011)$. Compared with the Non-SHPT patients, SHPT patients had a significantly lower $Y(p=0.012)$ and $\lambda(p=0.006)$.

\section{Network Efficiency}

Compared with healthy controls, only the SHPT patients had a significantly lower Eloc $(p=0.010)$. No group difference was found for Eg (Figure 2).

\section{Nodal characteristics}

\section{Identification of network hubs}

Network hub was defined if the nodal efficiency of a region was 1 SD greater than the average of the network. This study identified 12 hub nodes of the WM structural networks in the HC group and 14 hub nodes in both the SHPT and Non-SHPT groups. In three groups, 11 hub regions were identified in common, including bilateral supplementary motor area (SMA), the bilateral precentral gyrus (PreCG), bilateral precuneus (PCUN), right dorsolateral superior frontal gyrus (SFGdor), right middle frontal gyrus (MFG), right median cingulate and paracingulate gyri (DCG), left postcentral gyrus (PoCG) and right putamen (PUT). One hub region, the right middle temporal gyrus (MTG), was identified as a hub in the HC group. In addition, the left PUT was identified as a hub in both the SHPT and Non-SHPT groups. Two brain regions, the left middle frontal gyrus (MFG) and the right PoCG, were identified as hubs in the SHPT group but not in the Non-SHPT group. However, the left DCG and left middle occipital gyrus (MOG) were identified as hubs in the Non-SHPT group (Figure 3).

\section{Between-group differences in nodal efficiency}

We further analysis of group difference in nodal efficiency with the WM network (Figure 4, Table 3). Among the three groups, we found the regions which mainly distributed in the frontal and parietal cortices, including 5 frontal regions (bilateral SMA, left PreCG, left MFG and right rolandic operculum (ROL)), 3 parietal regions (bilateral PoCG and right SMG), 2 temporal regions (bilateral MTG) and 2 subcortical regions (right thalamus (THA) and right PUT) were most significantly decreased $(p<0.001)$. Post hoc tests showed that all these regions had a reduced efficiency in both the SHPT and Non-SHPT 
group compared with the HC ( $p<0.017)$, except the right PoCG in SHPT group. Moreover, 6 of these regions, including 2 frontal regions (left PreCG and left MFG), 2 parietal regions (bilateral PoCG), and 2 subcortical regions (right THA and right PUT) showed significantly reduced efficiency in the Non-SHPT group compared with the SHPT group $(p<0.017)$.

\section{Correlations between network metrics and neuropsychological tests and clinical data}

It was found that the iPTH and Lp were significantly negatively correlated with global cognition scores in the ESRD patients ( $r=-0.531, p=0.000 ; r=-0.316, p=0.028$, respectively). For the clinical data, we found that the iPTH level was significantly negatively correlated with $\mathrm{Y}(r=-0.299, p=0.039)$ and $\lambda(r=-0.372$, $p=0.009$ ) (Figure 5). For nodal efficiency, we conducted an analysis only for the nodes in the abnormal regions (the 12 regions in Table 3 ). Correlations between nodal efficiency and global cognition scores or clinical index in these regions were not found.

\section{Discussion}

The present study showed the topological alterations of WM networks in ESRD patients with SHPT by using DTI tractography and graph theory methods. The major findings are summarized as follows: (1) decreased Cp in the SHPT group, (2) lower Eloc for SHPT patients, (3) alterations in $y$ and $\lambda$ between the two patient groups, (4) alterations in nodal efficiency in the two patient groups compared with HC; and (5) a correlation between network metrics and neuropsychological tests and clinical data. These results will help us in understanding the neurophysiological mechanisms in ESRD patients with and without SHPT from a network perspective.

WM networks among the three groups displayed a typical small-world organization, although $\mathrm{y}$ and $\lambda$ were different between the SHPT and Non-SHPT group, which were in line with previous studies in healthy people[29, 30]. However, several topological properties, including $\mathrm{Cp}$ and Eloc, altered significantly between SHPT group and HC group. The fact that the WM network in SHPT patients had a lower Cp compared to healthy controls indicated ineffective information transmission in SHPT patients. From the perspective of network efficiency, we demonstrated a lower Eloc in SHPT patients compared to healthy controls, which suggests a loss of short-range connections throughout the brain network, likely attributable to changes in the modularized information processing ability and fault tolerance in the information transfers of the network[31]. We will continue to study this issue in the future for more convincing evidence.

Of the 12 brain regions identified as hubs from healthy controls, 11 brain regions were found in all three groups. We found both the SHPT and Non-SHPT group had added two more brain regions as hubs, the left MFG and the right PoCG, and the left DCG and MOG, respectively. On the other hand, the right MTG was no longer a hub in both the SHPT and Non-SHPT groups. These findings suggest that increased frontal-parietal interconnectivity and frontal-occipital interconnectivity in SHPT and Non-SHPT groups, which can be explained an increased susceptibility of these regions to ESRD patients, potentially due to the concentration of hubs in densely interconnected local networks [32]. The MTG is involved in several 
cognitive processes such as language and semantic memory processing and multimodal sensory integration[33-36]. Hubs play an important role in global information transfer and seem to be more vulnerable, since a stronger connection between hub regions leads to a higher probability of damage[37]. On the other hand, this result indicates that the weakened hub, likely relates to the cognitive impairment in the ESRD patients.

The spatial distribution of impaired brain regions by nodal efficiency was mainly located in the frontal and parietal cortices in ESRD patients. The node with the greatest disruption was the right THA, which is an intermediary for information processing, and the anterior and mediodorsal nucleus of THA influence memory storage and retrieval, respectively[38]. Frontal-parietal cortex lesions are considered to cause impairment of attention function, such as working memory, visuo-spatial attention and attention shift[3941]. Some previous studies have demonstrated that ESRD patients have neuropsychological problems such as attention, learning and memory, speed and decision making[42, 43]. Moreover, ESRD patients had significantly decreased cerebral blood flow in the frontal cortex and thalamus in a perfusion study[44]. In addition, several functional MRI studies have consistently shown regional homogeneity (ReHo) and functional connectivity (FC) are decreased in the frontal and parietal cortex in ESRD patients[45, 46]. In short, the concurrent abnormalities found in functional studies and our disrupted WM connection in the same brain region could be specific metabolic brain disorders in ESRD patients.

We first found that the MoCA scores were negatively associated with Lp, and the iPTH level was also negatively correlated with small-world organization by two indexes ( $\gamma$ and $\lambda$ ) in ESRD patients. The smallworldness indicates an optimal balance between local specialization and global integration. The Lp and small-worldness changes reflect a possible disruption of WM connections in ESRD patients with increased iPTH levels. However, we did not find any relationships between neuropsychological tests and the clinical data with the global network measures in the SHPT and Non-SHPT group, which may be attributable to the small sample size.

There are several limitations of this study that should be addressed. Firstly, we define the WM network edges which is insufficient in resolving fiber crossing by using deterministic tractography. Secondly, we only constructed WM structural networks, and a combination of the functional and structural network are needed in future studies. Third, we only performed MoCA tests to evaluate global cognitive, and a detailed battery of neuropsychological tests of cognitive function is needed in future studies. Fourth, the sample size is quite limited and should cautiously interpreting the results. Finally, we did not collect information of anemia in healthy controls, which may affect our results.

In conclusion, our results indicated that the brain structural connectome in ESRD patients with SHPT was more disrupted than in without SHPT patients, which was correlated with cognitive impairment. The iPTH level may a risk factor for cognitive impairment in ESRD patients, and the WM structure network has the potential to be a connectome-based biomarker for early detection of cognitive impairment.

\section{Declarations}




\section{Ethics approval and consent to participate}

This study was conducted in accordance with the 1964 Helsinki Declaration and its later amendments or comparable ethical standards. Participants provided written informed consent. This study was approved by the Ethics Committee of the Second Hospital of Anhui Medical University.

\section{Consent to publish}

Not applicable.

\section{Availability of data and materials}

The datasets used and/or analysed during the current study are available from the corresponding author on reasonable request.

\section{Competing interests}

The authors declare that they have no competing interests.

\section{Funding}

This work was supported by Anhui Provincial Public Linkage Projects (1604f0804025), University natural science research project of Anhui province (KJ2018A0201), Anhui Provincial General Medicine Clinical Scientific Projects (2016QK089) and Science research foundation of Anhui medical university (2017xkj037).

\section{Authors' Contributions}

Study concepts and design: Suisheng Zheng and Xijun Gong, Clinical data: Hanqiu Wu and Guiling Liu, Literature research: Yanqi Shan and Ru Zhao, Data analysis: Longsheng Wang and Liwei Zou, Statistical analysis: Liwei Zou and Fangbiao Tao, Manuscript preparation: Longsheng Wang and Liwei Zou, Manuscript editing: Fangbiao Tao

\section{Acknowledgements}

Not applicable.

\section{References}

1. Bugnicourt JM, Godefroy $\mathrm{O}$, Chillon JM, Choukroun G, Massy ZA: Cognitive disorders and dementia in CKD: the neglected kidney-brain axis. Journal of the American Society of Nephrology : JASN 2013, 24(3):353-363.

2. Liu ZH: Nephrology in china. Nature reviews Nephrology 2013, 9(9):523-528. 
3. Wang F, Yang C, Long J, Zhao X, Tang W, Zhang D, Bai K, Su Z, Gao B, Chu H et al: Executive summary for the 2015 Annual Data Report of the China Kidney Disease Network (CK-NET). Kidney international 2019, 95(3):501-505.

4. Kurella M, Chertow GM, Luan J, Yaffe K: Cognitive impairment in chronic kidney disease. Journal of the American Geriatrics Society 2004, 52(11):1863-1869.

5. Jorde R, Waterloo K, Saleh F, Haug E, Svartberg J: Neuropsychological function in relation to serum parathyroid hormone and serum 25 -hydroxyvitamin D levels. The Tromso study. Journal of neurology 2006, 253(4):464-470.

6. Seifter JL, Samuels MA: Uremic encephalopathy and other brain disorders associated with renal failure. Seminars in neurology 2011, 31(2):139-143.

7. Theofilou P: Quality of life in patients undergoing hemodialysis or peritoneal dialysis treatment. Journal of clinical medicine research 2011, 3(3):132-138.

8. Lee SY, Lee HJ, Kim YK, Kim SH, Kim L, Lee MS, Joe SH, Jung IK, Suh KY, Kim HK: Neurocognitive function and quality of life in relation to hematocrit levels in chronic hemodialysis patients. Journal of psychosomatic research 2004, 57(1):5-10.

9. Drueke TB, Locatelli F, Clyne N, Eckardt KU, Macdougall IC, Tsakiris D, Burger HU, Scherhag A, Investigators C: Normalization of hemoglobin level in patients with chronic kidney disease and anemia. The New England journal of medicine 2006, 355(20):2071-2084.

10. Singh AK, Szczech L, Tang KL, Barnhart H, Sapp S, Wolfson M, Reddan D, Investigators C: Correction of anemia with epoetin alfa in chronic kidney disease. The New England journal of medicine 2006, 355(20):2085-2098.

11. Chou FF, Chen JB, Hsieh KC, Liou CW: Cognitive changes after parathyroidectomy in patients with secondary hyperparathyroidism. Surgery 2008, 143(4):526-532.

12. Kim SM, Zhao D, Schneider ALC, Korada SK, Lutsey PL, Guallar E, Alonso A, Windham BG, Gottesman RF, Michos ED: Association of parathyroid hormone with 20-year cognitive decline: The ARIC study. Neurology 2017, 89(9):918-926.

13. Lee CB, Yu SH, Kim NY, Kim SM, Kim SR, Oh SJ, Jee SH, Lee JE: Association Between Coffee Consumption and Circulating Levels of Adiponectin and Leptin. Journal of medicinal food 2017, 20(11):1068-1075.

14. Pi HC, Xu YF, Xu R, Yang ZK, Qu Z, Chen YQ, Liu GL, Dong J: Cognitive Impairment and Structural Neuroimaging Abnormalities Among Patients with Chronic Kidney Disease. Kidney \& blood pressure research 2016, 41(6):986-996.

15. Kuriyama N, Mizuno T, Ohshima Y, Yamada K, Ozaki E, Shigeta M, Mitani S, Kondo M, Matsumoto S, Takeda $\mathrm{K}$ et al: Intracranial deep white matter lesions (DWLs) are associated with chronic kidney disease (CKD) and cognitive impairment: a 5-year follow-up magnetic resonance imaging (MRI) study. Archives of gerontology and geriatrics 2013, 56(1):55-60.

16. Luo S, Qi RF, Wen JQ, Zhong JH, Kong X, Liang X, Xu Q, Zheng G, Zhang Z, Zhang LJ et al: Abnormal Intrinsic Brain Activity Patterns in Patients with End-Stage Renal Disease Undergoing Peritoneal 
Dialysis: A Resting-State Functional MR Imaging Study. Radiology 2016, 278(1):181-189.

17. Kim JH, Choi NK, Kim SM: A Retrospective Study of Association between Peg-shaped Maxillary Lateral Incisors and Dental Anomalies. The Journal of clinical pediatric dentistry 2017, 41(2):150153.

18. Kalaitzidis RG, Karasavvidou D, Tatsioni A, Balafa O, Pappas K, Spanos G, Pelidou SH, Siamopoulos KC: Risk factors for cognitive dysfunction in CKD and hypertensive subjects. International urology and nephrology 2013, 45(6):1637-1646.

19. Bullmore E, Sporns O: Complex brain networks: graph theoretical analysis of structural and functional systems. Nature reviews Neuroscience 2009, 10(3):186-198.

20. Nasreddine ZS, Phillips NA, Bedirian V, Charbonneau S, Whitehead V, Collin I, Cummings JL, Chertkow $\mathrm{H}$ : The Montreal Cognitive Assessment, MoCA: a brief screening tool for mild cognitive impairment. Journal of the American Geriatrics Society 2005, 53(4):695-699.

21. Cui Z, Zhong S, Xu P, He Y, Gong G: PANDA: a pipeline toolbox for analyzing brain diffusion images. Frontiers in human neuroscience 2013, 7:42.

22. Tzourio-Mazoyer N, Landeau B, Papathanassiou D, Crivello F, Etard O, Delcroix N, Mazoyer B, Joliot M: Automated anatomical labeling of activations in SPM using a macroscopic anatomical parcellation of the MNI MRI single-subject brain. Neurolmage 2002, 15(1):273-289.

23. Zou L, Su L, Qi R, Bao F, Fang X, Wang L, Zhai Z, Li D, Zheng S: Abnormal topological organization in white matter structural networks in survivors of acute lymphoblastic leukaemia with chemotherapy treatment. Oncotarget 2017, 8(36):60568-60575.

24. Zhao T, Cao M, Niu H, Zuo XN, Evans A, He Y, Dong Q, Shu N: Age-related changes in the topological organization of the white matter structural connectome across the human lifespan. Human brain mapping 2015, 36(10):3777-3792.

25. Wang J, Wang X, Xia M, Liao X, Evans A, He Y: GRETNA: a graph theoretical network analysis toolbox for imaging connectomics. Frontiers in human neuroscience 2015, 9:386.

26. van den Heuvel MP, Sporns O: Network hubs in the human brain. Trends in cognitive sciences 2013, 17(12):683-696.

27. Sporns $\mathrm{O}$, Honey CJ, Kotter R: Identification and classification of hubs in brain networks. PloS one 2007, 2(10):e1049.

28. Khundrakpam BS, Lewis JD, Reid A, Karama S, Zhao L, Chouinard-Decorte F, Evans AC, Brain Development Cooperative G: Imaging structural covariance in the development of intelligence. Neurolmage 2017, 144(Pt A):227-240.

29. Gong G, He Y, Concha L, Lebel C, Gross DW, Evans AC, Beaulieu C: Mapping anatomical connectivity patterns of human cerebral cortex using in vivo diffusion tensor imaging tractography. Cerebral cortex 2009, 19(3):524-536.

30. Gong G, Rosa-Neto P, Carbonell F, Chen ZJ, He Y, Evans AC: Age- and gender-related differences in the cortical anatomical network. The Journal of neuroscience : the official journal of the Society for Neuroscience 2009, 29(50):15684-15693. 
31. Achard S, Bullmore E: Efficiency and cost of economical brain functional networks. PLoS computational biology 2007, 3(2):e17.

32. van den Heuvel MP, Sporns O: Rich-club organization of the human connectome. The Journal of neuroscience : the official journal of the Society for Neuroscience 2011, 31(44):15775-15786.

33. Siddarth P, Burggren AC, Eyre HA, Small GW, Merrill DA: Sedentary behavior associated with reduced medial temporal lobe thickness in middle-aged and older adults. PloS one 2018, 13(4):e0195549.

34. Deshpande AK, Tan L, Lu LJ, Altaye M, Holland SK: fMRI as a Preimplant Objective Tool to Predict Children's Postimplant Auditory and Language Outcomes as Measured by Parental Observations. Journal of the American Academy of Audiology 2018, 29(5):389-404.

35. Puschmann S, Thiel CM: Changed crossmodal functional connectivity in older adults with hearing loss. Cortex; a journal devoted to the study of the nervous system and behavior 2017, 86:109-122.

36. Nilakantan AS, Voss JL, Weintraub S, Mesulam MM, Rogalski EJ: Selective verbal recognition memory impairments are associated with atrophy of the language network in non-semantic variants of primary progressive aphasia. Neuropsychologia 2017, 100:10-17.

37. Crossley NA, Mechelli A, Scott J, Carletti F, Fox PT, McGuire P, Bullmore ET: The hubs of the human connectome are generally implicated in the anatomy of brain disorders. Brain : a journal of neurology 2014, 137(Pt 8):2382-2395.

38. Van der Werf YD, Jolles J, Witter MP, Uylings HB: Contributions of thalamic nuclei to declarative memory functioning. Cortex; a journal devoted to the study of the nervous system and behavior 2003, 39(4-5):1047-1062.

39. Smith R, Lane RD, Alkozei A, Bao J, Smith C, Sanova A, Nettles M, Killgore WDS: Maintaining the feelings of others in working memory is associated with activation of the left anterior insula and left frontal-parietal control network. Social cognitive and affective neuroscience 2017, 12(5):848-860.

40. Heinen K, Feredoes E, Ruff CC, Driver J: Functional connectivity between prefrontal and parietal cortex drives visuo-spatial attention shifts. Neuropsychologia 2017, 99:81-91.

41. Mastroberardino S, Santangelo V, Macaluso E: Crossmodal semantic congruence can affect visuospatial processing and activity of the fronto-parietal attention networks. Frontiers in integrative neuroscience 2015, 9:45.

42. Raphael KL, Wei G, Greene T, Baird BC, Beddhu S: Cognitive function and the risk of death in chronic kidney disease. American journal of nephrology 2012, 35(1):49-57.

43. Giang LM, Weiner DE, Agganis BT, Scott T, Sorensen EP, Tighiouart $H$, Sarnak MJ: Cognitive function and dialysis adequacy: no clear relationship. American journal of nephrology 2011, 33(1):33-38.

44. Fazekas G, Fazekas F, Schmidt R, Flooh E, Valetitsch H, Kapeller P, Krejs GJ: Pattern of cerebral blood flow and cognition in patients undergoing chronic haemodialysis treatment. Nuclear medicine communications 1996, 17(7):603-608.

45. Liang X, Wen J, Ni L, Zhong J, Qi R, Zhang LJ, Lu GM: Altered pattern of spontaneous brain activity in the patients with end-stage renal disease: a resting-state functional MRI study with regional homogeneity analysis. PloS one 2013, 8(8):e71507. 
46. Zheng G, Wen J, Zhang L, Zhong J, Liang X, Ke W, Kong X, Zhao T, He Y, Zuo X et al: Altered brain functional connectivity in hemodialysis patients with end-stage renal disease: a resting-state functional MR imaging study. Metabolic brain disease 2014, 29(3):777-786.

\section{Tables}

Table 1. Cortical and subcortical regions of interest defined in the study. 
Index Regions

$(1,2)$

$(3,4)$

Precental gyrus

Superior frontal gyrus, SFGdor dorsolateral

$(5,6)$

Superior frontal gyrus, ORBsup orbital part

$(7,8) \quad$ Middle frontal gyrus $\quad$ MFG

$(9,10)$

Middle frontal gyrus, orbital part

$(11,12) \quad$ Inferior frontal gyrus, IFGoperc opercular part

$(13,14) \quad$ Inferior frontal gyrus, IFGtriang triangular part

$(15,16) \quad$ Inferior frontal gyrus, ORBinf orbital part

$(17,18) \quad$ Rolandic operculum ROL

$(19,20)$ Supplementary motor SMA area

$(21,22) \quad$ Olfactory cortex

OLF

SFGmed

$(23,24)$

Superior frontal gyrus, medial

$(25,26) \quad$ Superior frontal gyrus, ORBsupmed medial orbital

$(27,28) \quad$ Gyrus rectus

REC

$(29,30) \quad$ Insula

INS

ACG

Anterior cingulate
paracingulate gyri

$(33,34)$

Median cingulate and paracingulate gyri

$(35,36) \quad$ Posterior cingulate gyrus

$(37,38) \quad$ Hippocampus

HIP

DCG

$(79,80) \quad$ Heschl gyrus

$(81,82)$

$(63,64)$ Supramarginal gyrus SMG

$(65,66) \quad$ Angular gyrus

$(67,68) \quad$ Precuneus

$(69,70) \quad$ Paracentral lobule

$(71,72) \quad$ Caudate nucleus

$(73,74) \quad$ Lenticular nucleus,

$(75,76)$ Lenticular
pallidium

$(77,78) \quad$ Thalamus

PCG

$(83,84)$
Superior temporal gyrus putamen

Lenticular nucleus,

THA

LING

SOG

MOG

IOG

FFG

PoCG

SPG

IPL

ANG

PCUN

PCL

CAU

PUT

PAL

Temporal pole: superior temporal gyrus

$(39,40) \quad$ Parahippocampal PHG

$(85,86) \quad$ Middle temporal gyrus MTG 
gyrus

\begin{tabular}{llllll}
\hline$(41,42)$ & Amygdala & AMYG & $(87,88)$ & $\begin{array}{l}\text { Temporal pole: middle } \\
\text { temporal gyrus }\end{array}$ & TPOmid \\
\hline$(43,44)$ & $\begin{array}{l}\text { Calcarine fissure and } \\
\text { surrounding cortex }\end{array}$ & CAL & $(89,90)$ & $\begin{array}{l}\text { Inferior temporal } \\
\text { gyrus }\end{array}$ & ITG \\
\hline$(45,46)$ & Cuneus & CUN & & & \\
\hline
\end{tabular}

Table 2. Demographic characteristics of study participants.

\begin{tabular}{lllll}
\hline Characteristics & SHPT $(\mathrm{n}=25)$ & Non-SHPT(n=25) & Healthy Controls $(\mathrm{n}=25)$ & $P$-value \\
\hline Age (year) & $42.20 \pm 7.53$ & $42.95 \pm 6.39$ & $41.80 \pm 7.15$ & $0.98^{\mathrm{a}}$ \\
Gender $(\mathrm{M} / \mathrm{F})$ & $14 / 11$ & $15 / 10$ & $12 / 13$ & $0.69^{\mathrm{b}}$ \\
Education(year) & $9.36 \pm 2.86$ & $9.44 \pm 3.23$ & $11.12 \pm 2.54$ & $0.06^{\mathrm{a}}$ \\
Disease duration(month) & $40.76 \pm 18.28$ & $38.44 \pm 16.84$ & - & $0.64^{\mathrm{c}}$ \\
Urea(mmol/L) & $26.14 \pm 7.08$ & $26.01 \pm 8.06$ & - & $0.95^{\mathrm{c}}$ \\
Creatinine( $\mu \mathrm{mol} / \mathrm{L})$ & $962.96 \pm 212.19$ & $914.48 \pm 274.11$ & - & $0.49^{\mathrm{c}}$ \\
Uric acid $(\mu \mathrm{mol} / \mathrm{L})$ & $447.04 \pm 38.42$ & $463.00 \pm 52.40$ & - & $0.52^{\mathrm{d}}$ \\
Calcium(mmol/L) & $2.37 \pm 0.33$ & $2.25 \pm 0.20$ & - & $0.14^{\mathrm{c}}$ \\
Phosphate $(\mathrm{mmol} / \mathrm{L})$ & $1.26 \pm 0.12$ & $1.27 \pm 0.10$ & - & $0.74^{\mathrm{c}}$ \\
Vitamin D $(\mathrm{ng} / \mathrm{ml})$ & $8.75 \pm 4.62$ & $11.14 \pm 4.09$ & - & $0.06^{\mathrm{c}}$ \\
iPTH $(\mathrm{pg} / \mathrm{mL})$ & $1401 \pm 721.06$ & $35.68 \pm 11.01$ & - & $0.00^{\mathrm{d}}$ \\
MoCA(score) & $22.92 \pm 2.22$ & $24.96 \pm 1.59$ & $28.00 \pm 1.22$ & $0.00^{\mathrm{a}^{*}}$ \\
\hline
\end{tabular}

Note: a $p$ value from one-way analysis of variance (ANOVA), b $p$ value from chi-square test, $\mathrm{c} p$ value from $\mathrm{t}$ test, $\mathrm{d} p$ value from Mann-Whitney $\mathrm{U}$ test. * means the post hoc analysis showed significant group difference between SHPT and Healthy controls. \# means the post hoc analysis showed significant group difference between Non-SHPT and Healthy controls

Table 3. Brain regions with significant group effects in nodal efficiency among SHPT, Non-SHPT patients and healthy controls. 


\begin{tabular}{|c|c|c|c|c|}
\hline Regions & $\begin{array}{l}\text { F value of } \\
\text { ANOVA(p value) }\end{array}$ & $\begin{array}{l}\text { p value of post hoc } \\
\text { test }\end{array}$ & & \\
\hline & & $\begin{array}{l}\text { SHPT versus } \\
\text { healthy controls }\end{array}$ & $\begin{array}{l}\text { Non-SHPT versus } \\
\text { healthy controls }\end{array}$ & $\begin{array}{l}\text { SHPT versus } \\
\text { Non-SHPT }\end{array}$ \\
\hline THA.R & $26.51(0.000)$ & 0.000 & 0.000 & 0.003 \\
\hline SMA.L & $22.74(0.000)$ & 0.000 & 0.000 & 0.341 \\
\hline SMA.R & $22.66(0.000)$ & 0.000 & 0.000 & 0.363 \\
\hline MTG.R & $22.09(0.000)$ & 0.000 & 0.000 & 0.039 \\
\hline PreCG.L & $19.20(0.000)$ & 0.002 & 0.000 & 0.004 \\
\hline PoCG.L & $17.44(0.000)$ & 0.005 & 0.000 & 0.004 \\
\hline SMG.R & $16.67(0.000)$ & 0.001 & 0.000 & 0.035 \\
\hline MFG.L & $16.36(0.000)$ & 0.008 & 0.000 & 0.004 \\
\hline ROL.R & $15.57(0.000)$ & 0.000 & 0.000 & 0.097 \\
\hline MTG.L & $14.56(0.000)$ & 0.000 & 0.000 & 0.285 \\
\hline PoCG.R & $14.14(0.000)$ & 0.044 & 0.000 & 0.002 \\
\hline PUT.R & $13.99(0.000)$ & 0.009 & 0.000 & 0.012 \\
\hline
\end{tabular}

Figures 


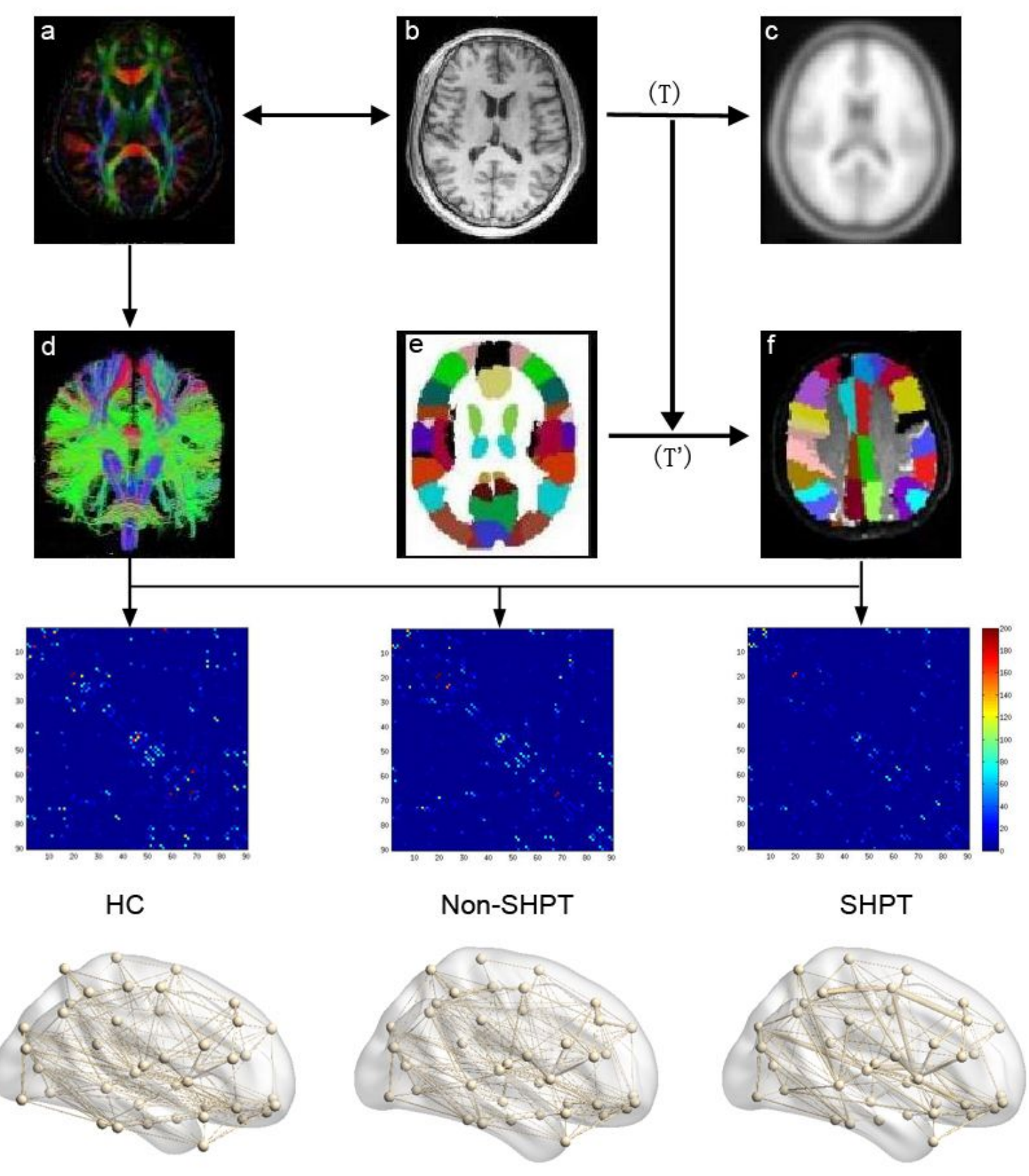

\section{Figure 1}

Flowchart for the construction of WM structural network by DTI. 1) The rigid coregisteration from T1weighted image (b) to the DTI native space (a). 2) The nonlinear registration from the resultant T1 image to the ICBM152 T1 template (c) in the MNI space resulting a transformation matrix (T). 3) The application of the inverse transformation $\left(T^{\prime}\right)$ to the AAL template in the MNI space (e), resulting in the subjectspecific AAL mask in the DTI native space (f). 4) The whole brain WM fibers were reconstructed (d) by DTI 
deterministic tractography method. 5) The fiber number (FN) weighted networks of each subject were created by computing FN that connected each pair of brain regions. The matrices and 3D representations (lateral view) of the mean WM structural network of each group are shown in the bottom panel. The nodes and edges were mapped onto the cortical surfaces by BrainNet viewer software. HC, healthy controls, Non-SHPT, end-stage renal disease (ESRD) without secondary hyperparathyroidism (SHPT) patients, SHPT, ESRD with SHPT patients.
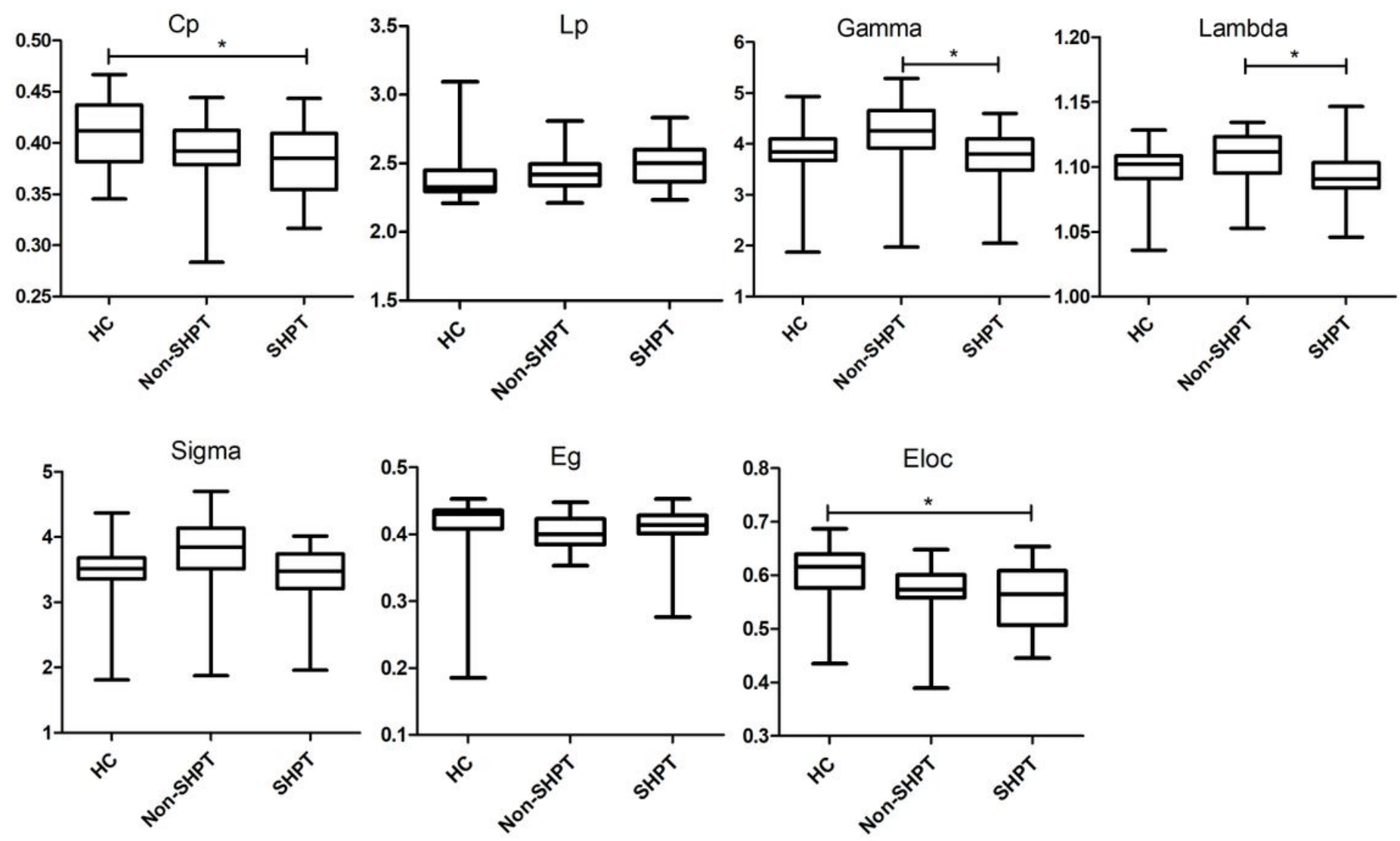

\section{Figure 2}

Boxplot shows the difference in global topological measures among the three group (medical, 50th percentile values, minimum and maximum). Significant differences were observed in $\mathrm{Cp}$ and Eloc between HC and SHPT group. Significant group effects were found $y$ and $\lambda$ between Non-SHPT and SHPT group. ${ }^{*} \mathrm{P}<0.017$. 
$\mathrm{L}$
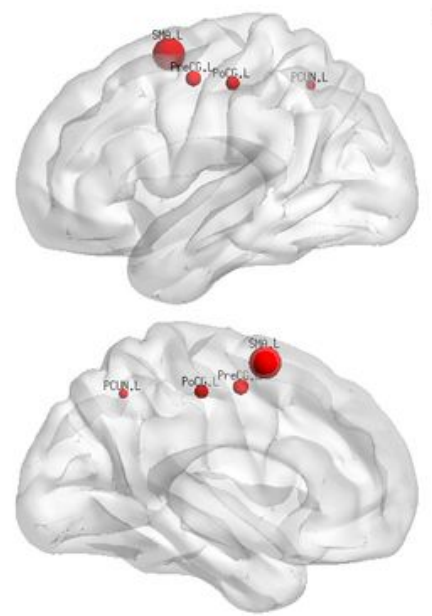

$\mathrm{HC}$

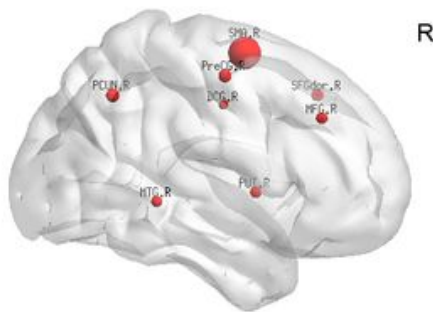

L
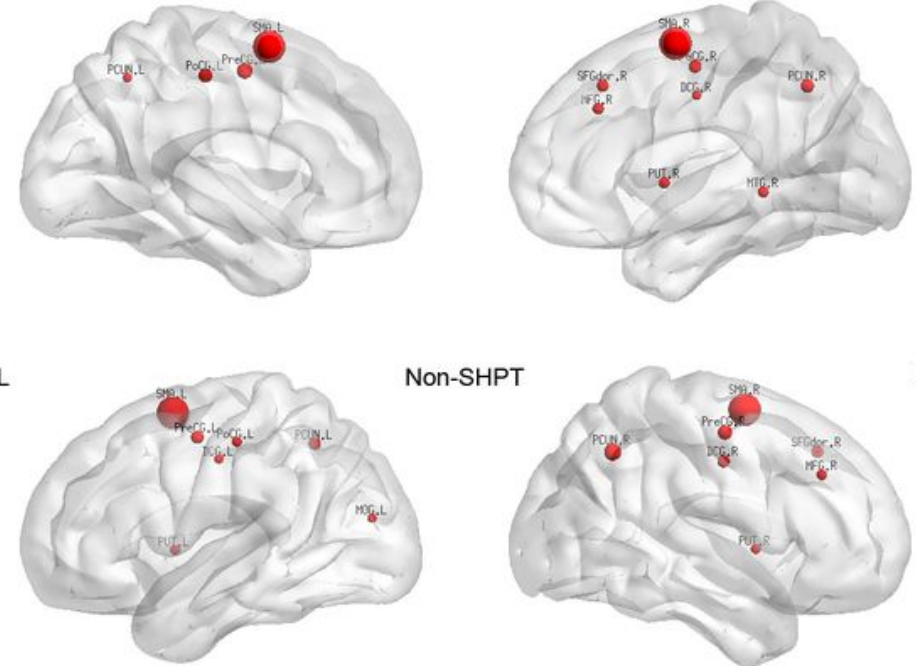

Non-SHPT

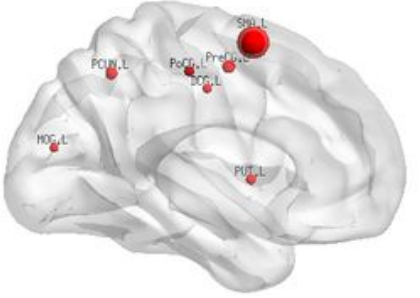

L

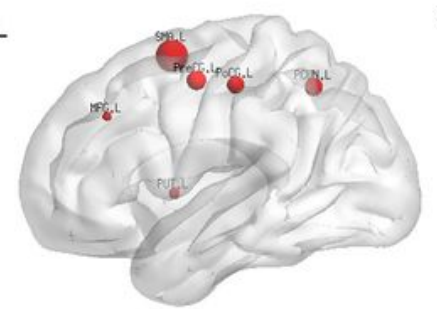

SHPT

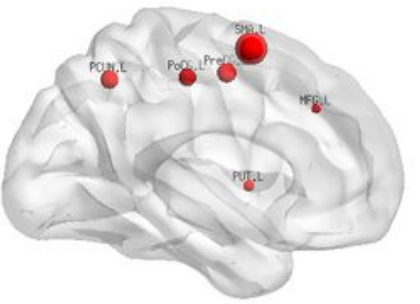

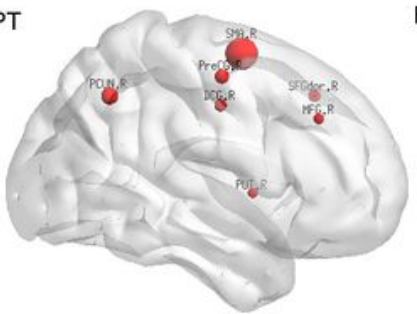

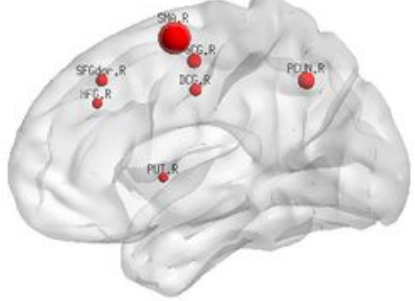

$\mathrm{R}$

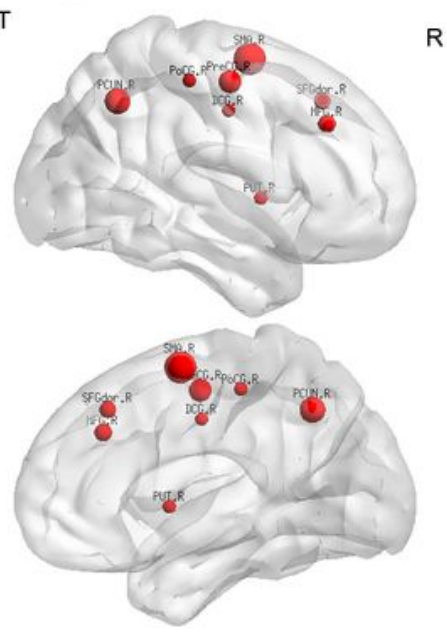

Figure 3

The global network hubs with nodal efficiency in HC, Non-SHPT and SHPT group. HC, healthy control, Non-SHPT, end-stage renal disease (ESRD) without secondary hyperparathyroidism (SHPT) patients, SHPT, ESRD with SHPT patients. 
$\mathbf{L}$
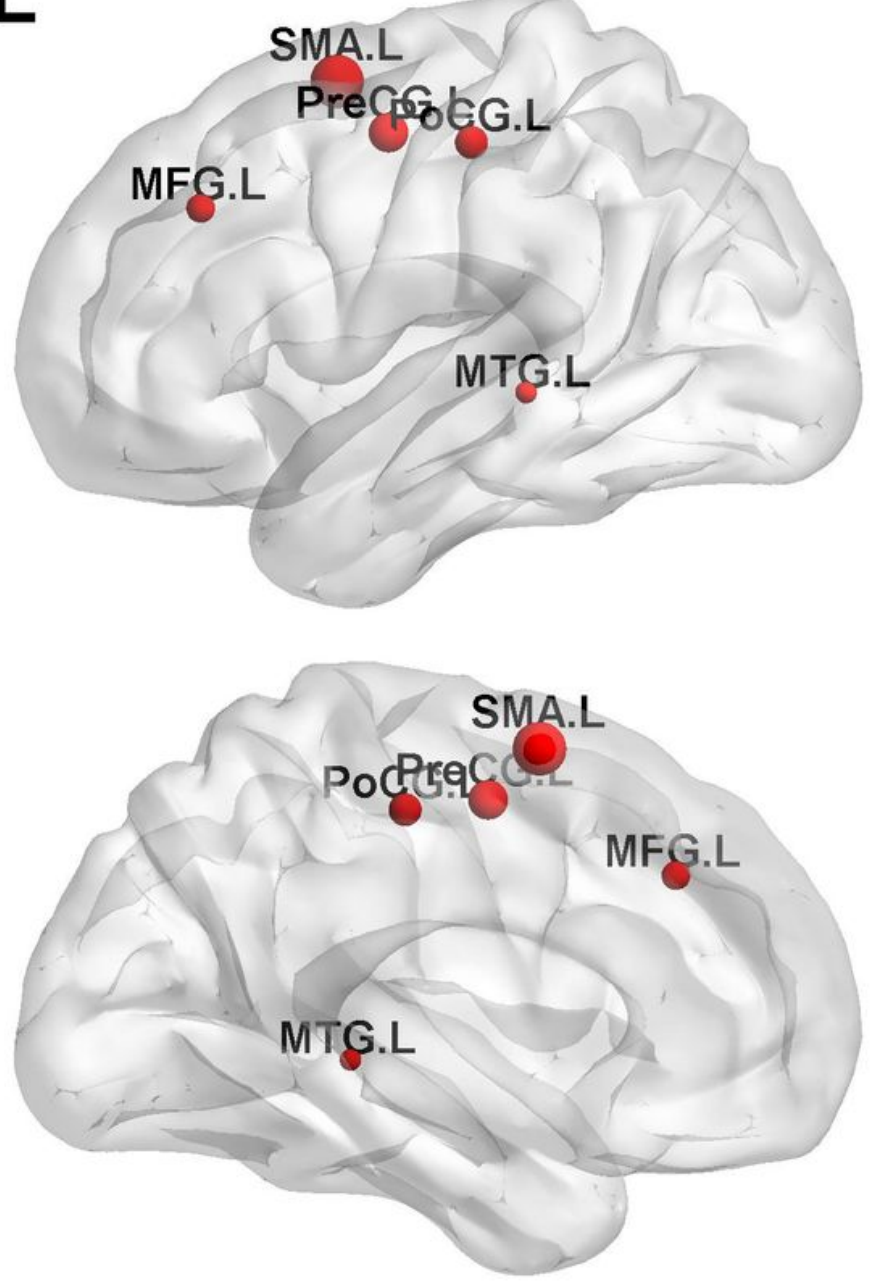

$\mathbf{R}$
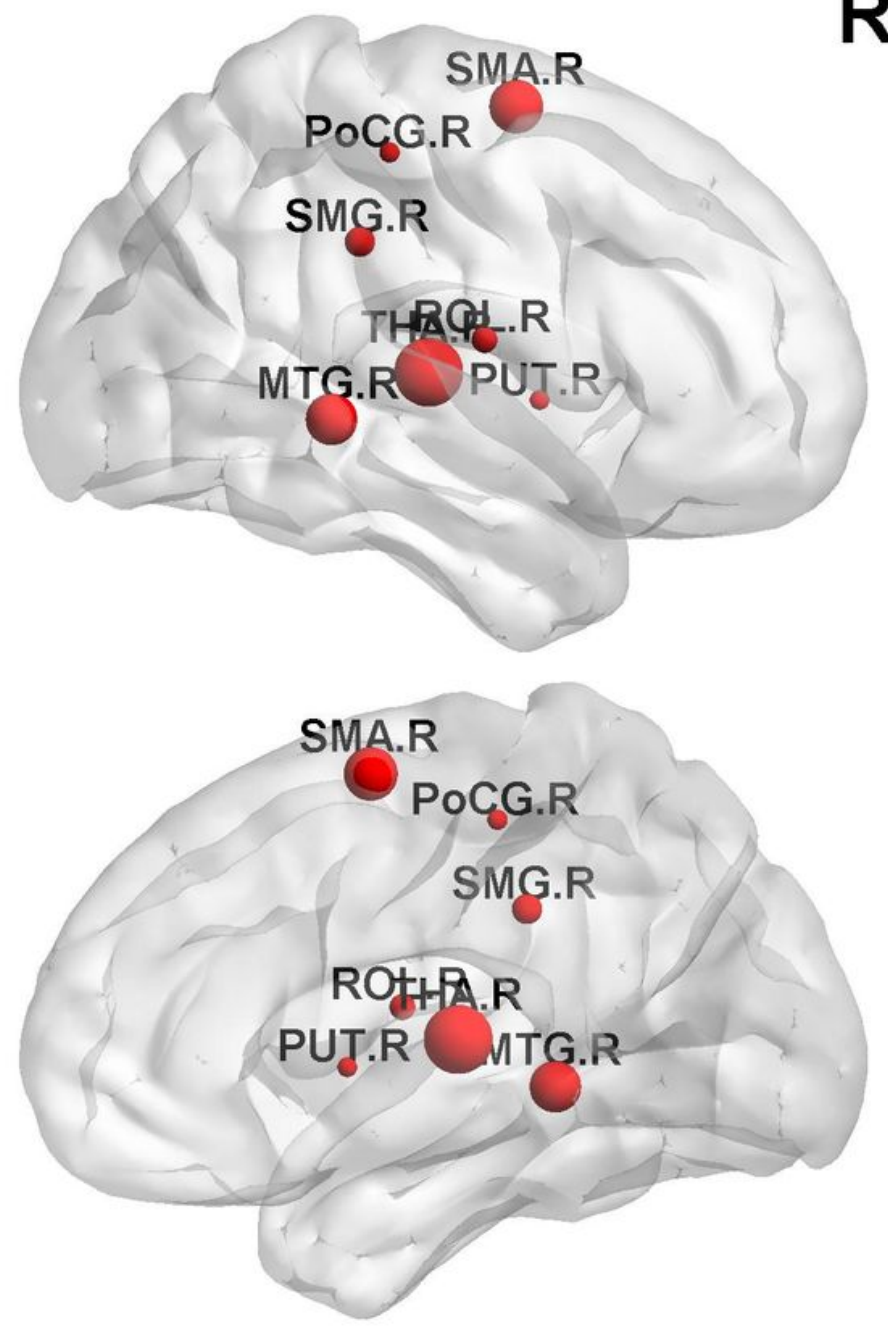

Figure 4

The distribution of brain regions with significant group difference in the nodal efficiency among three group. The node size represents the significance of between-group differences. 

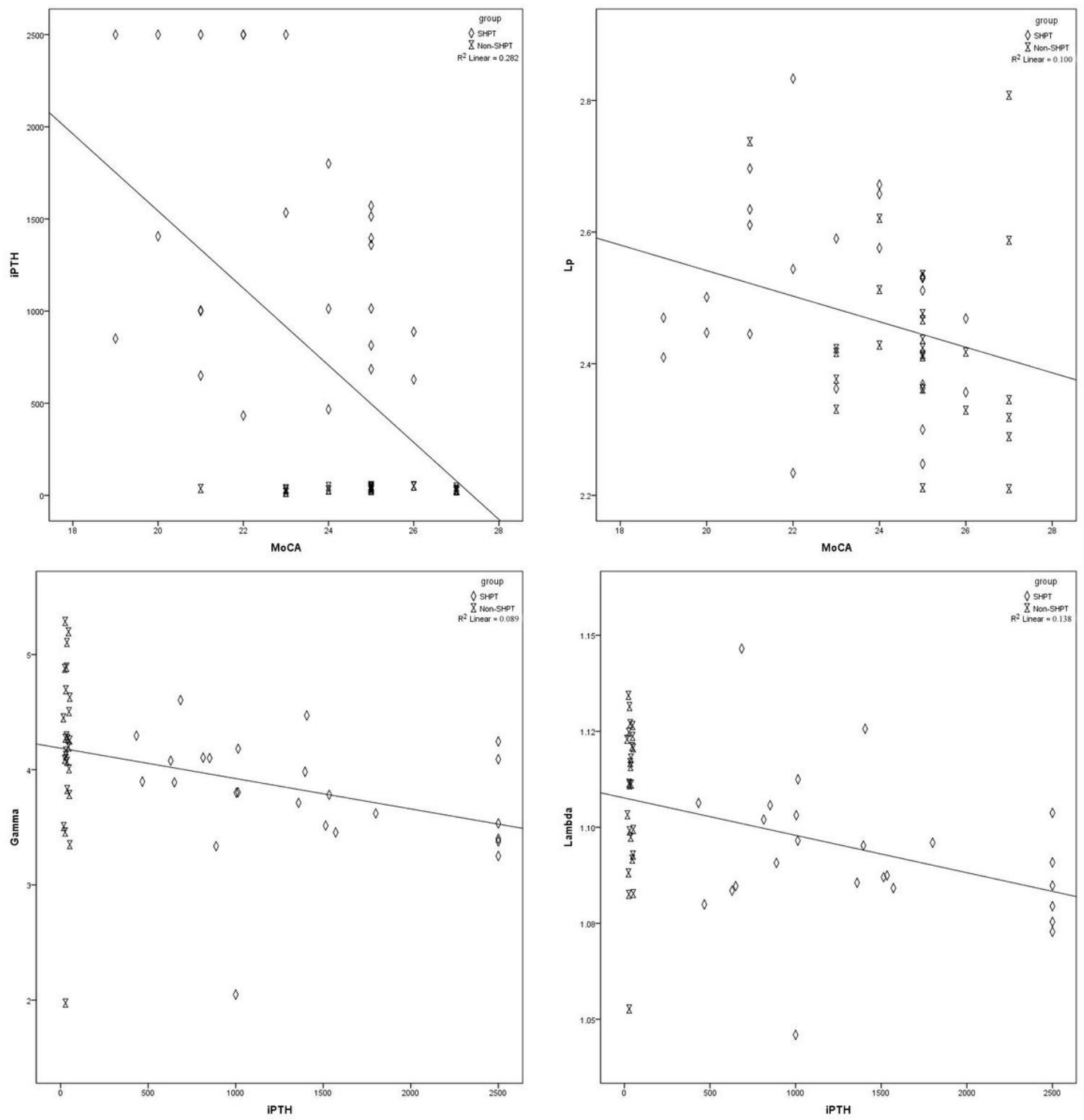

Figure 5

Scatterplots of correlation between network metrics and global cognition scores and clinical data.

Negative correlation between MoCA scores with iPTH level and Lp. Negative correlation between network topological property $(\mathrm{Y}$ and $\lambda$ ) and iPTH level.

Supplementary Files 
This is a list of supplementary files associated with this preprint. Click to download.

- supplement1.pdf 